JOURNAL OF

SYMPLECTIC GEOMETRY

Volume 1, Number 2, 253-267, 2002

\title{
New smooth counterexamples to the Hamiltonian Seifert conjecture
}

\author{
ELY KERMAN ${ }^{1}$
}

\begin{abstract}
We construct a new aperiodic symplectic plug and hence new smooth counterexamples to the Hamiltonian Seifert conjecture in $\mathbb{R}^{2 n}$ for $n \geq 3$. In other words, we describe an alternative procedure, to those of V.L. Ginzburg [Gi1, Gi2] and M. Herman [Her], for producing smooth Hamiltonian flows, on symplectic manifolds of dimension at least six, which have compact regular level sets that contain no periodic orbits. The plug described here is a modification of those built by Ginzburg. In particular, we use a different "trap" which makes the necessary embeddings of this plug much easier to construct.
\end{abstract}

\section{Introduction.}

The Poincaré recurrence theorem suggests that there are periodic orbits on every compact level set of a Hamiltonian flow. Indeed, there are now many theorems which establish the existence of periodic orbits for a variety of level sets and symplectic manifolds, see e.g., [FHV, Ho, HV, Ke, LT, Vi]. However, there is also a small set of examples of Hamiltonian flows which have compact regular level sets that contain no periodic orbits. These examples must be accounted for by any existence theorem and also serve to determine their limits.

The most important of these aperiodic flows are defined on the standard symplectic $\mathbb{R}^{2 n}$, for $n \geq 3$, and were first constructed, independently and in different ways, by V.L. Ginzburg [Gi1, Gi2] and M. Herman [Her]. More precisely, smooth Hamiltonian flows with aperiodic level sets were built for $n>3$ in [Gi1] and [Her], a $C^{3-\epsilon}$-example for $n=3$ was also defined in [Her], and finally a $C^{\infty}$-example for $n=3$ was constructed in [Gi2]. Among other things, these flows illustrate that the "almost existence" results of Hofer, Zehnder [HZ] and Struwe [St] are in some sense optimal. For a thorough discussion of the relevance of these examples to the various existence

\footnotetext{
${ }^{1}$ This work was supported by an NSERC postdoctoral fellowship.
} 
theorems, as well as a list of all the (previously) known Hamiltonian flows without periodic orbits, the reader is referred to the surveys [Gi3] and [Gi4].

In building their examples, both Ginzburg and Herman follow a general procedure due to Wilson [Wi], which uses the concept of an aperiodic plug to create aperiodic flows (see $\S 1.1$ ). However, they each extend this procedure to the Hamiltonian category in a different way. In particular, Herman symplectizes the plugs of Wilson [Wi] and Harrison [Ha], while Ginzburg builds a new type of plug entirely within the symplectic framework (see Definition 2.1). In this paper, we modify Ginzburg's constructions to obtain a comparatively simple symplectic plug and hence a new procedure for building smooth aperiodic Hamiltonian flows on $\mathbb{R}^{2 n}$ with $n \geq 3$. This plug is built around a different trap which makes the necessary embeddings of it much easier to construct. For example, in the most difficult case when $n=3$, we require only standard symplectic embedding theorems, whereas in [Gi2] it is necessary for Ginzburg to improve the dimensional constraints for some symplectic embedding results of M. Gromov [Gr1, Gr2].

In the remainder of this section, we briefly recall Wilson's strategy for producing aperiodic flows and discuss how it was used to find counterexamples to the Seifert conjecture. We also describe a Hamiltonian version of the Seifert conjecture as well as the motivation behind our choice of a different trap. In the second section, we recall Ginzburg's extension of Wilson's procedure to Hamiltonian flows. We then construct our new symplectic plug in the third section of the paper.

\subsection{Counterexamples to the Seifert and Hamiltonian Seifert conjectures.}

One of the most well known conjectures concerning the existence of periodic orbits is the Seifert conjecture. It asserts that every nonvanishing vector field on $S^{3}$ must have a closed trajectory. Since it was posed (asked) by Seifert in 1950 [Se], three counterexamples to this conjecture have been constructed; first in the $C^{1}$-category by $\mathrm{P}$. Schweitzer $[\mathrm{ScP}]$, then in the $C^{3-\epsilon}$-category by J. Harrison [Ha], and ultimately in the $C^{\omega}$-category by K. Kuperberg [KuK1]. A volume preserving version of Schweitzer's flow was also built by G. Kuperburg in $[\mathrm{KuG}]$. For a review of these counterexamples see [KuK2] and [Gi3].

The Seifert conjecture can be extended to Hamiltonian flows in a number of ways [Gi3]. Consider the Hamiltonian flow defined by a smooth function $H$ on a symplectic manifold. For the purposes of this paper, the Hamiltonian Seifert conjecture will be the assertion that there exists a periodic orbit of 
this flow on every compact regular level set of $H$. Just as its namesake, this conjecture is known to be false. The constructions of Ginzburg, Herman and those defined here, can be used to produce counterexamples on any symplectic manifold of dimension at least six. Indeed, these aperiodic Hamiltonian flows can be glued into any Darboux chart. However, the conjecture remains open for $\mathbb{R}^{4}$. It also continues to merit study because these rare examples of aperiodic level sets may help to illuminate the poorly understood boundary between existence and nonexistence theorems, (see $\S 3.4$ of [Gi4]).

All of the counterexamples mentioned above are based on Wilson's construction of aperiodic flows in [Wi]. Starting with a manifold $W$, of dimension $k$, and a vector field $X$, in a specific class, his idea is simply to make special local alterations to $X$ which destroy all periodic orbits. More precisely, suppose that $X$ has a periodic orbit which passes through the point $w \in W$. One would like to modify $X$ near $w$ so that

1) for the resulting vector field, the trajectory through $w$ is no longer closed

2) no new periodic orbits are created

3) the new vector field is still in the desired class.

To accomplish this, Wilson introduced the notion of an aperiodic plug. This consists of a compact product manifold $P=[-1,1] \times N$, a carefully constructed vector field $V$ on $P$ which is parallel to $[-1,1]$ on $\partial P$, and an embedding $j: P \rightarrow \mathbb{R}^{k}$ such that $d j(V)$ is constant near $\partial P$. With this, the dynamical system defined by $V$ on $P$ can be inserted, via $j$, into a flow box around $w$. This results in a new local flow, and the properties of $j$ and $V$ on $\partial P$ imply that the only trajectories that are altered are those which enter $j(P)$ through $j(\{-1\} \times N)$. Once they are inside the image of $P$, these trajectories behave like trajectories of $V$ whose flow has been designed to accomplish the goals above. For example, with the exception of K. Kuperberg's plug, the first goal is achieved as follows. The flow of $V$ is built so that some point $(-1, \tilde{p}) \in\{-1\} \times N$ asymptotically approaches an aperiodic orbit on an invariant submanifold $M$ that lies in the interior of $P$. The insertion of $P$ into the flow box is then chosen so that $(-1, \tilde{p})$ gets mapped to $w$. As a result, the new trajectory through $w$ becomes trapped in the image of $P$ and, because of its asymptotic behavior, is no longer closed. The submanifold $M$ together with its inherited flow is called the trap (or core) of the plug. 
Remark 1.1. The remarkable feature of K. Kuperberg's plug is that, instead of using a known aperiodic flow as a trap, she creates an entirely new aperiodic dynamical system in dimension three (see [KuK1, Gh]).

Every plug which uses a trap to destroy periodic orbits is defined on a thickening of the trap's manifold $M$, i.e., $P \simeq M \times D^{l}$ for some closed ball $D^{l} \subset \mathbb{R}^{l}$. Hence, in order to construct the embedding $j$ for such a plug one must first find a suitable embedding for $M$. In [Wi], Wilson uses the irrational flow on a two-dimensional torus as a trap. Around this, he constructs a smooth plug which can be embedded in a flow box of dimension at least four. Schweitzer then constructs a $C^{1}$-plug in $[\mathrm{ScP}]$ which can be embedded in a flow box of dimension three and with this he obtains the first counterexamples to the original Seifert conjecture. The trap which enables Schweitzer to do this is a punctured two-dimensional torus with a flow that is trivial near the boundary and contains the (aperiodic) closure of an (aperiodic) orbit of the Denjoy flow in the interior. Unlike the complete torus, the punctured torus can be embedded into $\mathbb{R}^{3}$ in such a way that the standard normal vectors are everywhere parallel to a constant vector (see Figure 3. of $[\mathrm{ScP}]$ ). By thickening this embedding along the normal directions, Schweitzer is then able to appropriately embed his plug into $\mathbb{R}^{3}$.

What allows Schweitzer to use the punctured torus is the fact that the Denjoy flow on $\mathbb{T}^{2}$ has a proper minimal set that contains no closed orbits. (Note that the Denjoy flow is $C^{1}$ and that no $C^{2}$-flow on any surface admits such a minimal set $[\mathrm{ScA}]$.) His crucial observation can be summarized as follows :

The flow on the trap only needs to be aperiodic on an invariant closed subset.

The strength of this simple statement lies in the fact that we know of very few examples of totally aperiodic flows on manifolds of small dimension.

In [Gi1] and [Gi2], Ginzburg uses a trap consisting of the horocycle flow on $S T^{*} \Sigma$, the unit cotangent bundle of a surface $\Sigma$ with constant negative curvature. This flow is not only aperiodic on all of $S T^{*} \Sigma$, but is also minimal [Hed]. In order to use this trap to obtain the counterexample for $n=3$, it was necessary for Ginzburg to prove a new symplectic embedding theorem in [Gi2]. Using Schweitzer's observation above, our choice of a trap is a relatively simple flow on $\mathbb{T}^{3}$ which is only aperiodic on an invariant subset. For this trap, the construction of the necessary embeddings is much simpler. Unfortunately, this choice does not allow us to reduce to the case $n=2$. Most likely, the construction of a counterexample for this final case (if it is 
even possible!) will require, as in [KuK1], the creation of an entirely new type of Hamiltonian dynamics.

Acknowledgments. I am deeply grateful to Viktor Ginzburg for many encouraging and helpful comments. In particular, the relative simplicity of the construction presented here owes much to one of his suggestions.

\section{Symplectic plugs and aperiodic Hamiltonian flows.}

In this section we describe how the special nature of a Hamiltonian flow allows one to reformulate the Hamiltonian Seifert conjecture and to use a different type of plug to produce counterexamples.

We begin by considering the existence of periodic orbits for a nonvanishing vector field $X$ on an odd-dimensional manifold $W$, and we note that it is possible to rephrase this question given an additional geometric structure. Recall that a two-form is said to be maximally nondegenerate if its kernel is everywhere one-dimensional. Let us assume that we can find such a form $\sigma$ on $W$ so that $X(w)$ spans $\operatorname{ker} \sigma(w)$ for all $w \in W$. Then, instead of looking for periodic orbits of $X$, we may equivalently look for smooth maps $\gamma: S^{1} \rightarrow W$ for which $\dot{\gamma}(t)$ spans $\operatorname{ker} \sigma(\gamma(t))$ for all $t \in[0,1]$. Such maps are called closed characteristics of $\sigma$ on $W$ and we will identify those having the same image. If $\gamma$ is instead defined on some interval, i.e., the image isn't a loop, it will just be called a characteristic of $\sigma$.

Now, let $H$ be a smooth function on a symplectic manifold $(Q, \Omega)$ and let $W$ be a compact regular level set of $H$. The corresponding Hamiltonian flow is generated by the Hamiltonian vector field $X_{H}$ defined by the equation

$$
i_{X_{H}} \Omega=d H
$$

This vector field is tangent to $W$ and nonvanishing there. It is also easy to verify that $\sigma=\left.\Omega\right|_{W}$ is maximally nondegenerate and that $X_{H}$ spans $\operatorname{ker} \sigma$. From the discussion above, we see that the Hamiltonian Seifert conjecture can be reformulated as a statement which asserts the existence of closed characteristics for certain maximally nondegenerate two-forms. In particular, the conjecture concerns those forms which are obtained by restricting a symplectic form to a hypersurface in a symplectic manifold. With this version of the conjecture in mind, Ginzburg introduced the following notion of a symplectic plug as a tool to construct counterexamples.

Definition 2.1 ([Gi1]). A symplectic plug for dimension $2 n(n>2)$ is a product manifold $P=[-1,1] \times N^{2 n-2}$, equipped with an exact maximally 
nondegenerate two-form $\omega$, and embeddings $j: P \rightarrow \mathbb{R}^{2 n-1}$ and $J: P \rightarrow \mathbb{R}^{2 n}$ such that the following conditions hold:

(P.1) $\operatorname{ker} \omega$ is vertical (parallel to $[-1,1])$ near $\partial P$.

(P.2) there exists a point $(-1, \tilde{p}) \in\{-1\} \times N$ such that any characteristic of $\omega$ through this point does not pass through $\{1\} \times N$.

(P.3) a characteristic through $(-1, p) \in\{-1\} \times N$ which meets $\{1\} \times N$ does so at $(1, p)$.

(P.4) $\omega$ has no closed characteristics in $P$.

(P.5) $j^{*} \Omega_{2 n-2}=\omega$ near $\partial P$, where $\Omega_{2 n-2}$ is the pullback of the canonical symplectic form on $\mathbb{R}^{2 n-2}$ via the projection $\mathbb{R}^{2 n-1} \rightarrow \mathbb{R}^{2 n-2}$.

(P.6) for a given $\delta>0$ we have $J: P \rightarrow[-\delta, \delta] \times \mathbb{R}^{2 n-1}$ and $J^{*} \Omega_{2 n}=$ $\omega$. Moreover, if we let $\tilde{j}$ be the map $j$ with its range identified with $\{0\} \times \mathbb{R}^{2 n-1} \subset \mathbb{R}^{2 n}$, then $J=\tilde{j}$ near $\partial P$ and $J$ is isotopic to $\tilde{j}$ relative to $\partial P$.

Remark 2.2. It is perhaps unnecessary to include the embedding $j$ in this definition. However, as described below, this map allows a symplectic plug to be used for the slightly different purpose of destroying closed characteristics for any maximally nondegenerate two-form. It also helps to clarify the construction of the embedding $J$. Indeed, here and in [Gi1] the map $J$ is of the form $J=f \circ j$ where $f: \mathbb{R}^{2 n-1} \rightarrow \mathbb{R}^{2 n}$. Again, $j$ is used to destroy a closed characteristic while the map $f$ now ensures that this is done without disturbing the symplectic structure. When the domain of $f$ is identified with $\{0\} \times \mathbb{R}^{2 n-1} \subset \mathbb{R}^{2 n}$ we will denote it by $\tilde{f}$ so that $J=\tilde{f} \circ \tilde{j}$.

Symplectic plugs were constructed for $n>3$ in [Gi1] and for $n=3$ in [Gi2]. In [Gi1], Ginzburg also describes how a symplectic plug for dimension $2 n$ can be used to obtain counterexamples to the Hamiltonian Seifert conjecture in $\mathbb{R}^{2 n}$. We recall this procedure here.

As a first step, we show that a symplectic plug can be used to destroy a closed characteristic of a maximally nondegenerate two-form $\sigma$ on an odddimensional manifold $W^{2 n-1}$. In fact, assuming that $\sigma$ has only a finite number of closed characteristics, we will construct from it a new maximally nondegenerate two-form having none. Let $w \in W$ be a point in the image of one of the closed characteristics of $\sigma$ and choose a small open neighborhood of $w$ which does not meet the others. This neighborhood can be chosen to 
be diffeomorphic to an open ball $B^{2 n-1} \subset \mathbb{R}^{2 n-1}$ in such a way that $\sigma$ gets identified with $\Omega_{2 n-2}$. This is a version of a flow box around $w$ since the characteristics of $\Omega_{2 n-2}$ in $\mathbb{R}^{2 n-1}$ are all straight. We can then use $j$ to insert $(P, \omega)$ into this neighborhood so that $(-1, \tilde{p})$ gets mapped to $w$. Let $\sigma^{\prime}$ be the form which equals $\sigma$ outside of $j(P)$ and $\omega$ inside. By (P.1) and (P.5), we see that $\sigma^{\prime}$ is well-defined, smooth and maximally nondegenerate. Properties (P.2), (P.3) and (P.4) then imply that the characteristics of $\sigma^{\prime}$ through $w$ are no longer closed and that $\sigma^{\prime}$ has no new closed characteristics. We have thus succeeded in finding a maximally nondegenerate two-form on $W$ with exactly one less closed characteristic than $\sigma$. Applying this procedure to the other closed characteristics we see that the existence of symplectic plugs for $n \geq 3$ implies the following theorem.

Theorem 2.3 ([Gi1, Gi2]). Assume that $\operatorname{dim} W \geq 5$ and $\sigma$ has a finite number of closed characteristics. Then there exists a closed maximally nondegenerate two-form $\sigma^{\prime}$ which is homotopic to $\sigma$ and has no closed characteristics.

Remark 2.4. Two closed maximally nondegenerate two-forms are said to be homotopic if they can be joined by a one parameter family of such forms all lying in the same cohomology class. The existence of the homotopy in Theorem 2.3 follows from one between $\omega$ and a two-form $\omega_{1}$ which has a vertical kernel and satisfies $j^{*} \Omega_{2 n-2}=\omega_{1}$. This homotopy is defined in Remark 3.3 and is not a necessary feature for a symplectic plug.

We can now use the embedding $J$ to extend the previous theorem to one which applies to a hypersurface $W$ in a symplectic manifold $\left(Q^{2 n}, \Omega\right)$. Assume that $\left.\Omega\right|_{W}$ has a finite number of closed characteristics and let $w \in W$ be a point on one of them. There exists a neighborhood $U$ of $w$ in $Q$ such that $U$ doesn't meet the other closed characteristics on $W$ and $U$ is a symplectic flow box. In other words, $U$ is diffeomorphic to an open ball $B^{2 n} \subset \mathbb{R}^{2 n}=\mathbb{R} \times \mathbb{R}^{2 n-1}$ in such a way that the intersection of $B^{2 n}$ with $\{0\} \times \mathbb{R}^{2 n-1}$ corresponds to $U \cap W$, the form $\Omega$ gets identified with $\Omega_{2 n}$ and $\left.\Omega\right|_{U \cap W}$ gets identified with $\Omega_{2 n-2}$ on $\{0\} \times \mathbb{R}^{2 n-1}$. We can then use $J$ (corresponding to a sufficiently small $\delta$ ) to insert $(P, \omega)$ into $U$ so that $J(\partial P) \subset U \cap W$ and $(-1, \tilde{p})$ gets mapped to $w$. Let $W^{\prime}$ be the hypersurface which is equal to $W$ outside of $U$ and agrees with the image of $\mathrm{J}$ inside of $U$. We claim that $\left.\Omega\right|_{W^{\prime}}$ has exactly one less closed characteristic than $\left.\Omega\right|_{W}$. To see this, we view the insertion of $(P, \omega)$, by $J=\tilde{f} \circ \tilde{j}$, as a two step process (see Remark 2.2). The primary insertion uses $\tilde{j}$ to map $(P, \omega)$ into 
$U \cap W$. As above, this yields a new form $\Omega^{\prime}$ on $W$ with exactly one less closed characteristic than $\left.\Omega\right|_{W}$. The map $\tilde{f}$ then deforms $W$ into $W^{\prime}$ so that $\Omega^{\prime}=\tilde{f}^{*} \Omega$. In particular, $\tilde{f}$ takes the characteristics of $\Omega^{\prime}$ through $w \in W$ to the characteristics of $\left.\Omega\right|_{W^{\prime}}$ through $w \in W^{\prime}$, which can therefore never be closed. Repeating this process for the other closed characteristics we get;

Theorem 2.5 ([Gi1, Gi2]). Assume that $\operatorname{dim} Q \geq 6$ and that $\left.\Omega\right|_{W}$ has a finite number of closed characteristics. Then there exists a smooth hypersurface $W^{\prime} \subset Q, C^{0}$-close and isotopic to $W$, such that $\left.\Omega\right|_{W^{\prime}}$ has no closed characteristics.

Let $W$ be an irrational ellipsoid in $\left(\mathbb{R}^{2 n}, \Omega_{2 n}\right)$, i.e., a level set of a function of the form $H(z)=\sum_{j=1}^{n} a_{j}\left\|z_{j}\right\|^{2}$ where the $a_{j}$ are independent over $\mathbb{Q}$. Then $W$ contains only $n$ closed characteristics and can be modified, as above, to obtain a concrete example of a smooth hypersurface (regular level set) with no closed characteristics (periodic orbits), see Corollary 3.3 and Corollary 3.4 of [Gi3].

\section{A new symplectic plug.}

We now build a new symplectic plug for $n=3$. This construction can easily be extended to obtain plugs for $n>3$ as well.

\subsection{The trap.}

As a trap, we will use the constant-speed geodesic flow of the flat metric on the two-dimensional torus. This is described in terms of a Hamiltonian flow as follows. The symplectic manifold is $\left(T^{*} \mathbb{T}^{2}, d \lambda\right)$, the cotangent bundle of the two-dimensional torus equipped with its canonical symplectic structure. With respect to the (global) coordinates $\left\{\theta_{1}, \theta_{2}, p_{1}, p_{2}\right\}$ on $T^{*} \mathbb{T}^{2}=\mathbb{T}^{2} \times \mathbb{R}^{2}$, the Hamiltonian is the standard kinetic energy function defined by

$$
\begin{aligned}
H: T^{*} \mathbb{T}^{2} & \rightarrow \mathbb{R} \\
\left(\theta_{1}, \theta_{2}, p_{1}, p_{2}\right) & \mapsto \frac{1}{2}\left(p_{1}^{2}+p_{2}^{2}\right) .
\end{aligned}
$$

It is easy to check that the Hamiltonian vector field of $H$ has only base components and that its flow on the torus $\mathbb{T}^{2} \times\left\{\left(p_{1}, p_{2}\right)\right\}$ is the constant linear flow with slope $\frac{p_{2}}{p_{1}}$. Our trap will be the level set $M=H^{-1}\left(R^{2}\right)$ together with its inherited flow. If we let $S$ be the dense subset of $M$ consisting of the points whose last two components, $\left(p_{1}, p_{2}\right)$, are rationally independent, 
then each torus $\mathbb{T}^{2} \times\left\{\left(p_{1} . p_{2}\right)\right\} \subset S$ is a closed invariant aperiodic subset of $M$. Alternatively, our trap can be described as a manifold $M$ which is diffeomorphic to the three dimensional torus and is equipped with an exact maximally nondegenerate two-form $d \eta=\left.d \lambda\right|_{M}$. Moreover, $M$ contains a dense subset $S$ such that every characteristic of $d \eta$ which starts in $S$ stays in $S$ and is not closed.

Switching to polar coordinates $\left(p_{1}, p_{2}\right) \rightarrow\left(r, \theta_{3}\right)$ we see that $\bar{\theta}=$ $\left(\theta_{1}, \theta_{2}, \theta_{3}\right)$ is a set of coordinates on $M$ and

$$
\eta=R \cos \left(\theta_{3}\right) d \theta_{1}+R \sin \left(\theta_{3}\right) d \theta_{2} .
$$

We will call the angle $\theta_{3}$ irrational if it corresponds to a rationally independent pair $\left(p_{1}, p_{2}\right)$.

\subsection{The plug.}

Let $N=[-\epsilon, \epsilon] \times M$ so that $P=[-1,1] \times[-\epsilon, \epsilon] \times M$. We label the coordinates for the two intervals by $t$ and $x$, respectively. Consider the two-form on $P$ defined by

$$
\omega=d\left(A\left(\theta_{3}, x, t\right) \eta+B(x, t) d t\right)
$$

for smooth functions $A$ and $B$ of the indicated coordinates.

Claim 3.1. Let the functions $A$ and $B$ have the following properties:

(A.1) $A>0$.

(A.2) $A_{x}^{\prime} \geq 0$, with equality only at $\left(\tilde{\theta}_{3}, 0, \pm \frac{1}{2}\right)$ where $\tilde{\theta}_{3}$ is a fixed irrational angle. These are also the only two critical points of $A$.

(A.3) $A=1+x$ near $\partial P$.

(A.4) $A$ is even in $t$.

(B.1) $B=0$ near $\partial P$.

(B.2) $B=x$ near $\left(0, \pm \frac{1}{2}\right)$.

(B.3) $B$ is odd in $t$.

Then, $\omega$ is maximally nondegenerate and (P.1), (P.2), (P.3) and (P.4) hold.

Remark 3.2. Functions satisfying the conditions in the claim are easily constructed. 
Proof. Treating $\omega$ as a family of skew-symmetric matrices parameterized by $P$, it is easy to check that the coefficient of the linear term in the characteristic polynomial of $\omega\left(\theta_{1}, \theta_{2}, \theta_{3}, x, t\right)$ is

$$
R^{4} A^{2}\left(A_{x}^{\prime 2}+A_{t}^{\prime 2}\right)+R^{2}{B_{x}^{\prime 2}}^{2}\left(A^{2}+A_{\theta_{3}}^{\prime 2}\right) .
$$

Clearly, $\omega$ is maximally nondegenerate if and only if this coefficient is never zero. By properties (A.1) and (A.2), the first term only vanishes at $\left(\tilde{\theta}_{3}, 0, \pm \frac{1}{2}\right)$. At these points the second term becomes

$$
R^{2} B_{x}^{\prime 2} A^{2}
$$

which does not equal zero by property (B.2). Thus, $\omega$ is maximally nondegenerate.

Near the boundary of $P$ we have

$$
\omega=d x \wedge \eta+(1+x) d \eta .
$$

Since $\eta$ is nondegenerate on ker $d \eta$, we see that $\frac{\partial}{\partial t}$ spans $\operatorname{ker} \omega$ near $\partial P$. Hence, $\omega$ has property (P.1).

In order to check (P.2), (P.3) and (P.4), we consider a nonvanishing vector field $V$ on $P$ such that $V$ spans $\operatorname{ker} \omega$. We choose $V$ so that it equals $\frac{\partial}{\partial t}$ near $\partial P$ and we recall that any characteristic of $\omega$ is equivalent (after some reparameterization) to a trajectory of $V$.

It is straightforward to check that

$$
\omega \wedge \omega \wedge d t=R^{2} A A_{x}^{\prime} \mu
$$

where $\mu$ is the standard volume form with respect to our coordinates on $P$. Since $i_{V} \omega=0$, it follows that the $t$-component of $V, V_{t}$, satisfies

$$
V_{t} \omega \wedge \omega=R^{2} A A_{x}^{\prime} i_{V} \mu .
$$

This equation implies that $V_{t}$ vanishes only when $R^{2} A A_{x}^{\prime}$ does. Hence, by property (A.2), $V_{t}$ equals zero along the two-dimensional tori $\mathbb{T}_{ \pm}^{2}$ defined by $\left(\theta_{3}, x, t\right)=\left(\tilde{\theta}_{3}, 0, \pm \frac{1}{2}\right)$, respectively. By continuity and our choice of $V$ near $\partial P$, we also see that $V_{t}$ is strictly positive away from these tori. On $\mathbb{T}_{ \pm}^{2}$ we have,

$$
\omega=A\left(\tilde{\theta}_{3}, 0, \pm \frac{1}{2}\right) d \eta+d x \wedge d t
$$

Hence, $V$ is tangent to both tori and lies in the kernel of $d \eta$ on each. This means that the flows of $V$ on $\mathbb{T}_{-}^{2}$ and $\mathbb{T}_{+}^{2}$ are both conjugate to the irrational 
flow for the angle $\tilde{\theta}_{3}$. Let $L^{-}\left(\mathbb{T}_{-}^{2}\right)$ be the negative limit set of $\mathbb{T}_{-}^{2}$ under the flow of $V$. The previous observations then imply that the set

$$
(\{-1\} \times N) \cap L^{-}\left(\mathbb{T}_{-}^{2}\right)
$$

is nonempty and that all the points inside it asymptotically approach the aperiodic flow on $\mathbb{T}_{-}^{2}$. Consequently, the trajectories of $V$ through all of these points are trapped in $P$ and property (P.2) holds.

At this point, it is also easy to see that none of the trajectories of $V$ are closed and hence (P.4) is satisfied. This is because $V_{t}$ is nonnegative and so any periodic orbit of $V$ would have to lie on either $\mathbb{T}_{+}^{2}$ or $\mathbb{T}_{-}^{2}$, where we know the flow of $V$ is aperiodic.

To verify (P.3), we look at the map $\psi: P \rightarrow P$ which sends $t \rightarrow-t$ and acts as the identity on the $N$-component. By properties (A.4) and (B.3), it is possible to choose $V$ so that

$$
d \psi(V)=-V .
$$

Consider a trajectory of $V$ (characteristic of $\omega$ ) which starts at a point $(-1, p) \in\{-1\} \times N$ and exits $P$ through $\{1\} \times N$. The $t$-component $V_{t}$ can not vanish along this trajectory since it would then be trapped in one of the internal tori $\mathbb{T}_{ \pm}^{2}$ and could never exit $P$. Consequently, the (anti-)symmetry of $V$ described above implies that as the trajectory progresses from $t=0$ to $t=1$ it retraces, in reverse, the progress it made along the $N$-component from $t=-1$ to $t=0$. The trajectory must then exit $P$ at $(1, p)$ and property (P.3) is satisfied.

In order to complete the construction of our plug we must find embeddings $j: P \rightarrow \mathbb{R}^{5}$ and $J: P \rightarrow \mathbb{R}^{6}$ which satisfy (P.5) and (P.6). First, consider the restriction of $\omega$ to

$$
\{-1\} \times N \simeq[-\epsilon, \epsilon] \times M .
$$

We call this restriction $\rho$ and note that

$$
\rho=d x \wedge \eta+(1+x) d \eta
$$

This form restricts to the hypersurface $\{0\} \times M$ as $d \eta$. Notice that $[-\epsilon, \epsilon] \times M$ can also be considered as a (closed) neighborhood of $M$ in $T^{*} \mathbb{T}^{2}$ and recall that the canonical symplectic form $d \lambda$ also restricts to $M$ as $d \eta$. Using Weinstein's Extension theorem [We], it is easy to show that two symplectic forms which agree on a compact oriented hypersurface are equivalent in a 
neighborhood of that hypersurface, see [MS, Exercise 3.35, p.102]. Hence, for sufficiently small $\epsilon$ there exists a symplectomorphism

$$
\phi_{1}:([-\epsilon, \epsilon] \times M, \rho) \rightarrow(\mathcal{N}(M), d \lambda),
$$

where $\mathcal{N}(M)$ is an open neighborhood of $M$ in $T^{*} \mathbb{T}^{2}$. Next, for sufficiently small $R$ (and $\epsilon$ ), we can assume that $\mathcal{N}(M)$ lies in an arbitrarily small neighborhood of the zero section in $T^{*} \mathbb{T}^{2}$. Invoking the Lagrangian Neighborhood theorem, there exists a symplectomorphism

$$
\phi_{2}:(\mathcal{N}(M), d \lambda) \rightarrow\left(\mathbb{R}^{4}, \Omega_{4}\right)
$$

onto a neighborhood surrounding a Clifford torus in $\mathbb{R}^{4}$. Composing $\phi_{1}$ and $\phi_{2}$ we get a symplectomorphism

$$
\phi:([-\epsilon, \epsilon] \times M, \rho) \rightarrow\left(\mathbb{R}^{4}, \Omega_{4}\right) .
$$

We now define the embedding $j$ by

$$
\begin{aligned}
j: P=[-1,1] \times N & \rightarrow \mathbb{R} \times \mathbb{R}^{4} \\
(t, p) & \mapsto(t, \phi(p)) .
\end{aligned}
$$

Since $\omega=\rho$ near all of $\partial P, j$ satisfies (P.5).

Remark 3.3. To form the homotopy discussed in Remark 2.4, we replace $A$ and $B$ in the definition of $\omega$ by the family of functions

$$
\begin{aligned}
& A_{\tau}=(1-\tau) A+\tau(1+x) \\
& B_{\tau}=(1-\tau) B .
\end{aligned}
$$

The resulting two-forms, $\omega_{\tau}$, are all maximally nondegenerate and by the construction of $j$ we also have $j^{*} \Omega_{4}=\omega_{1}$.

As noted in Remark 2.2, the embedding $J$ is of the form $J=f \circ j$ for some embedding $f: j(P) \subset \mathbb{R}^{5} \rightarrow \mathbb{R}^{6}$. For simplicity we will identify $j(P)$ with $P$. We then choose $f$ so that it maps $P$ into $\mathbb{R} \times P \subset \mathbb{R} \times \mathbb{R}^{5}$. On $\mathbb{R} \times P$ we have coordinates $(y, t, x, \bar{\theta})$. Recalling that $j^{*}\left(\Omega_{4}\right)=\rho$ we see that with respect to these coordinates

$$
\begin{aligned}
\left.\Omega_{6}\right|_{\mathbb{R} \times P} & =d y \wedge d t+\rho \\
& =d(y d t+(1+x) \eta) .
\end{aligned}
$$


Let $f: P \rightarrow \mathbb{R} \times P$ be defined by

$$
(t, x, \bar{\theta}) \mapsto(B, t, A-1, \bar{\theta})
$$

Property (A.2) ensures that $f$ is indeed an embedding. In addition, we may choose the function $B$ to be arbitrarily small so that given any $\delta>0$ the image of $f$ will lie in $(-\delta, \delta) \times \mathbb{R}^{5}$. Note also that $A$ and $B$ are equal to $1+x$ and 0 on $\partial P$, respectively, and are also isotopic to these functions. This implies that $J$ equals $\tilde{j}$ near $\partial P$ and is isotopic to $\tilde{j}$ relative to $\partial P$. Hence, we only need to prove that $f^{*} \Omega_{6}=\omega$. In fact, we can verify the stronger fact that

$$
f^{*}(\underbrace{y d t+(1+x) \eta}_{\alpha})=A \eta+B d t .
$$

To see this let $v \in T_{p} P$. Then

$$
\begin{aligned}
f^{*} \alpha(v) & =\alpha(f)[d f(v)] \\
& =(A \eta+B d t)[d f(v)] \\
& =(A \eta+B d t)[v]
\end{aligned}
$$

where the last equality holds because $f$ acts as the identity map on $t$ and $\bar{\theta}$. With this, the construction of our symplectic plug is complete.

\section{References.}

[FHV] A. Floer, H. Hofer and C. Viterbo, The Weinstein conjecture in $P \times \mathbb{C}^{l}$, Math. Z., 203 (1990), 469-482.

[Gh] E. Ghys, Construction de champs de vecteurs sans orbite périodique (d'après Krystyna Kuperberg), Sém. Bourbaki, 19931994, Astérisque, 227, Soc. Math. France, Paris, (1994), 283-307.

[Gi1] V.L. Ginzburg, An embedding $S^{2 n-1} \rightarrow \mathbb{R}^{2 n}, 2 n-1 \geq 7$ whose Hamiltonian flow has no periodic trajectories, IMRN, 2 (1995), 83-97.

[Gi2] V.L. Ginzburg, A smooth counterexample to the Hamiltonian Seifert conjecture in $\mathbb{R}^{6}$, IMRN, 13 (1997), 641-650.

[Gi3] V.L. Ginzburg, Hamiltonian dynamical systems without periodic orbits, Amer. Math Soc. Transl. (2), 196 (1999), 35-48. 
[Gi4] V.L. Ginzburg, The Hamiltonian Seifert conjecture: examples and open problems, to appear in 'Proceedings of the Third European Congress of Mathematics,' Preprint 2000; math.DG/0004020.

[Gr1] M. Gromov, A topological technique for the construction of solutions of differential equations and inequalities, Proc. Internat. Congr. Math. (Nice, 1970), 2, Gauthier-Villars, Paris, (1971), 221-225.

[Gr2] M. Gromov, Partial differential relations, Springer-Verlag, New York, 1986.

[Ha] J. Harrison, A $C^{2}$ counterexample to the Seifert conjecture, Topology, 27 (1988), 249-278.

[Hed] G.A. Hedlund, Fuschian groups and transitive horocycles, Duke Math. J., 2 (1936), 530-542.

[Her] M.-R. Herman, Examples of compact hypersurfaces in $\mathbb{R}^{2 p}, 2 p \geq 6$, with no periodic orbits, in 'Hamiltonian systems with three or more degrees of freedom,' C. Simo (Editor), NATO Adv. Sci. Inst. Ser. C, Math. Phys. Sci., 533, Kluwer Acad. Publ., Dordrecht, 1999.

[Ho] H. Hofer, Pseudoholomorphic curves in symplectizations with applications to the Weinstein conjecture in dimension three, Invent. Math., 114 (1993), 515-563.

[HV] H. Hofer and C. Viterbo, The Weinstein conjecture for cotangent bundles and related results, Ann. Scuola Norm. Sup. Pisa Cl. Sci. (4) 15(3) (1988), 411-445.

[HZ] H. Hofer and E. Zehnder, Periodic solution on hypersurfaces and a result by C. Viterbo, Invent. Math., 90 (1987), 1-9.

[Ke] E. Kerman, Periodic orbits of Hamiltonian flows near symplectic critical submanifolds, IMRN, 17 (1999), 953-969.

[KuG] G. Kuperberg, A volume-preserving counterexample to the Seifert conjecture, Comment. Math Helv., 71 (1996), 239-268.

[KuK1] G. Kuperberg, A smooth counterexample to the Seifert conjecture in dimension three, Ann. of Math. (2), 140 (1994), 723-732.

[KuK2] G. Kuperberg, Counterexamples to the Seifert conjecture, Proc. Internat. Congr. Math., II (Berlin 1998), 831-840. 
[LT] G. Liu and G. Tian, Weinstein conjecture and $G W$ Invariants, preprint, 1999.

[MS] D. McDuff and D. Salamon, Introduction to symplectic topology, Oxford University Press, New York, 1995.

[ScA] A generalization of a Poincaré-Bendixson theorem to closed twodimensional manifolds, Amer. J. Math., 85 (1963), 453-458.

$[\mathrm{ScP}]$ P.A. Schweitzer, Counterexamples to the Seifert conjecture and opening closed leaves of foliations, Ann. of Math. (2), 100 (1970), 229-234.

[Se] H. Seifert, Closed integral curves in 3-space and isotopic twodimensional deformations, Proc. Amer. Math. Soc., 1 (1950), 287302.

[St] M. Struwe, Existence of periodic solutions of Hamiltonian systems on almost every energy surface, Bol. Soc. Bras. Mat., 20 (1990), 49-58.

[Vi] C. Viterbo, A proof of the Weinstein conjecture in $\mathbb{R}^{2 n}$, Ann. Inst. H. Poincaré, Anal. Non Linéare, 4 (1987), 337-356.

[We] A. Weinstein, Lectures on symplectic manifolds, CBMS, 29, Reg. Conf. Ser. in Math., AMS, 1977.

[Wi] F. Wilson, On the minimal sets of non-singular vector fields, Ann. of Math. (2), 84 (1966), 529-536.

The Fields Institute

222 College Street, Toronto

ONTARIO M5T 3J1, CANADA

E-mail address: ekerman@fields.utoronto.ca 
